\title{
Distribuição ecológica de comunidades de macroalgas de ambientes lóticos do Parque Nacional de Itatiaia (RJ, MG), Brasil
}

\author{
ORLANDO NECCHI JÚNIOR ${ }^{1,2}$, LUIS H.Z. BRANCO ${ }^{1}$ e DANIEL N. SPEZAMIGLIO ${ }^{1}$
}

(recebido: 03 de maio de 2007; aceito: 21 de fevereiro de 2008)

\begin{abstract}
Ecological distribution of stream macroalgal communities from "Parque Nacional de Itatiaia", states of Minas Gerais and Rio de Janeiro, Brazil). Itatiaia National Park (INP) includes two kinds of Brazilian vegetation within its limits: Atlantic tropical rainforest (AF) and highland grassland (HG). Fourteen sites were sampled, seven in each region ( $\mathrm{AF}$ and $\mathrm{HG}$ ), in two seasons (summer and winter). Twenty nine species of macroalgae were surveyed, from which 15 in $\mathrm{AF}$ and 19 in $\mathrm{HG}$, with only five species in common. Species richness per sampling site ranged from 1 to $7(2.9 \pm 2.0)$, Shannon-Wiener diversity index $\left(H^{\prime}\right)$ from 0 to $0.94(0.24 \pm 0.26)$ and percent cover from 0 to $35 \%(14.0 \pm 12.5 \%)$. These values fitted within the range reported in previous studies on lotic macroalgae. Multiple regression analysis revealed that temperature variations accounted for $39.2 \%$ of abundance and $35.5 \%$ of species richness variations and also that $54.8 \%$ of diversity variations were explained by variations of Chemical Oxygen Demand (COD) and pH. Cluster analysis of macroalgal communities of INP did not group sites from the same regions (AF and HG). Principal Component Analysis clearly separated the sampling sites of the two regions, as well as of the two seasons (summer and winter), mostly influenced by temperature, shading, $\mathrm{pH}$, altitude, potassium and COD. The general patterns of patchy distribution and dominance by few species described for lotic macroalgal communities were corroborated by data from INP, suggesting that they seem to be universal for such communities.
\end{abstract}

Key-words - Atlantic rainforest, distribution, highland grassland, lotic ecosystem, macroalgae

RESUMO - (Distribuição ecológica de comunidades de macroalgas de ambientes lóticos do Parque Nacional de Itatiaia (RJ, MG), Brasil). O Parque Nacional de Itatiaia (PNI) inclui duas formações vegetais brasileiras em seus limites: floresta pluvial tropical atlântica (MA) e campos de altitude (CA). Foram amostrados 14 pontos, sete em cada formação vegetal, em duas estações do ano (verão e inverno). Foram encontradas 29 espécies de macroalgas, sendo 15 espécies em MA e 19 espécies em $\mathrm{CA}$, com apenas cinco espécies em comum. A riqueza de espécies por ponto de amostragem variou de 1 a $7(2,9 \pm 2,0)$, o índice de diversidade de Shannon-Wiener $\left(H^{\prime}\right)$ de 0 a $0,94(0,24 \pm 0,26)$ e a cobertura percentual de 0 a $35 \%(14,0 \pm 12,5 \%)$. Estes valores situaram-se dentro da amplitude reportada em trabalhos prévios sobre macroalgas lóticas. A análise de regressão múltipla revelou que as variações de temperatura explicaram 39,2\% da variação da abundância e 35,5\% da riqueza de espécies a ainda que 54,8\% da variação da diversidade foi explicada por Demanda Química de Oxigênio (DQO) e pH. Análise de grupamento das comunidades de macroalgas do PNI não agrupou pontos das mesmas regiões (MA e CA). Análise de Componentes Principais separou claramente os pontos amostrados das regiões de MA e CA, bem como das estações do ano estudadas (inverno e verão), influenciados mais fortemente pela temperatura, sombreamento, $\mathrm{pH}$, altitude, potássio e DQO. Os padrões gerais de distribuição em mosaico e dominância por poucas espécies descritos para comunidades de macroalgas lóticas foram corroborados pelos dados do PNI, sugerindo que parecem ser universais para tais comunidades.

Palavras-chave - campos de altitude, distribuição, ecossistema lótico, macroalga, mata atlântica

\section{Introdução}

Estudos relevantes sobre comunidades de macroalgas lóticas têm sido desenvolvidos em regiões boreais, temperadas ou tropicais de distintos continentes: Australásia (Biggs 1990, Biggs \& Price 1987, Entwisle 1989), Europa (Holmes \& Whitton 1981, Johansson 1982, John \& Moore 1985, Kawecka 1980, 1981, e vários

1. Universidade Estadual Paulista, Departamento de Zoologia e Botânica, Rua Cristóvão Colombo, 2265, 15054-000 São José do Rio Preto, SP, Brasil.

2._Autor para correspondência: orlando@ibilce.unesp.br exemplos em Whitton 1984), América do Norte (Sheath \& Burkholder 1985, Sheath \& Cole 1992, Sheath et al. 1986, 1989, 1996) e América do Sul (Branco \& Necchi Júnior 1996a, 1996b, 1997, 1998, Necchi Júnior et al. 1995, 1997, 2000a, 2003).

Investigações sobre a distribuição ecológica de comunidades de macroalgas lóticas são relativamente escassas, com poucas realizadas em regiões tropicais no mundo. Poucos trabalhos foram realizados enfocando regiões de florestas tropicais, podendo-se destacar: Sheath \& Cole (1992) para a América do Norte; Vis et al. (1994) para o arquipélago do Havaí; Mosisch \& Bunn (1997) para riachos da Austrália; e McClintic et al. (2003) para 
floresta pluvial tropical da Bolívia. No Brasil as florestas tropicais também têm sido pouco estudadas e as únicas publicações específicas sobre comunidades de macroalgas lóticas restringem-se a regiões de floresta tropical atlântica no Estado de São Paulo (Branco \& Necchi 1996a, 1996b, Necchi Júnior et al. 2000a), além do trabalho de Pascoaloto (2001) em riachos da floresta amazônica. Estes estudos evidenciaram alguns padrões gerais: a distribuição em mosaico das comunidades, dominância por poucas espécies e predominância de Chlorophyta em relação aos demais grupos de algas.

Estudos sobre a distribuição ecológica de comunidades de macroalgas lóticas em regiões montanhosas ou de alta latitude são igualmente esparsos. Sheath et al. (1996) e Sheath \& Müller (1997) apresentaram dados sobre macroalgas de águas correntes de regiões de tundra no Ártico e áreas montanhosas esparsas têm sido investigadas na Europa (Kawecka \& Eloranta 1987, Uehlinger 1991, Pfister 1992, 1993, Hieber et al. 2001, Medvedeva 2001), América do Norte (Wehr 1981, Ward 1986, 1994, Meegan \& Perry 1996, Vavilova \& Lewis Junior 1999, Gustina \& Hoffmann 2000), América do Sul (McClintic et al. 2003) e Ásia (Ormerod et al. 1994). No Brasil, a pesquisa em riachos de montanhas encontra-se ainda em estágio muito inicial. Alguns poucos trabalhos relatam aspectos descritivos de habitat e fauna bentônica (Callisto et al. 2001, Galdean et al. 2001), análises experimentais de enriquecimento de nutrientes sobre a comunidade perifítica (Mendes \& Barbosa 2002) ou descrevem dados limnológicos de algumas regiões montanhosas do Estado de Minas Gerais (Necchi Júnior et al. 2000b, 2003). Poucos trabalhos da literatura brasileira relatam a ocorrência de macroalgas em campos de altitude e foram realizados nos Parques Nacionais de Itatiaia e da Serra da Canastra. Bicudo \& Bicudo (1969) estudaram algas de ambiente lêntico, incluindo algumas macroalgas. Necchi Júnior et al. (2003) estudaram região de campos de altitude no Parque Nacional da Serra da Canastra, onde foram inventariadas a flora, a riqueza e a abundância de espécies das comunidades de macroalgas e a relação com as variáveis ambientais em uma área referente à bacia de drenagem do alto Rio São Francisco.

O presente trabalho teve como objetivos: 1) descrever as características da estrutura (composição, riqueza, abundância e diversidade) das comunidades de macroalgas das áreas de campos de altitude e de Mata Atlântica do Parque Nacional de Itatiaia; 2) comparar a estrutura de comunidades entre regiões e épocas do ano, analisando a existência ou não de padrões; 3 ) avaliar quais as variáveis ambientais mais influentes sobre os padrões da estrutura dessas comunidades.

\section{Material e métodos}

O Parque Nacional do Itatiaia (PNI) localiza-se em áreas dos Estados do Rio de Janeiro e Minas Gerais (22 $15^{\circ}$ $22^{\circ} 30^{\prime} \mathrm{S}, 44^{\circ} 30^{\prime}-44^{\circ} 45^{\prime} \mathrm{W}$ ) e abrange a parte mais elevada da Serra da Mantiqueira, tendo como ponto culminante o Pico das Agulhas Negras com 2.787 m (IBAMA 1994). Em sua parte mais baixa, a vegetação encontrada é tipicamente a floresta pluvial atlântica (Mata Atlântica, MA), que se modifica gradualmente com a elevação da altitude, sendo substituída pela típica vegetação de campos de altitude (CA) nas áreas mais altas (>1.650 m).

Foram realizadas amostragens em duas épocas do ano: no final do inverno ( 24 a 28 de setembro de 2002) e no final do verão (17 a 20 de março de 2003). Quatorze pontos de amostragem foram analisados dentro dos limites do PNI nas duas épocas (tabela 1, figura 1), setes deles localizados na região de menor altitude (MA), enquanto que os outros sete, na região mais alta $(\mathrm{CA})$.

As comunidades de macroalgas foram analisadas nos pontos de amostragens quanto à composição, riqueza e abundância de espécies através da técnica de transeção (Holmes \& Whitton 1981, Sheath \& Burkholder 1985, Krebs 1989, Necchi Júnior et al. 2000a). A transeção (faixa) consistiu
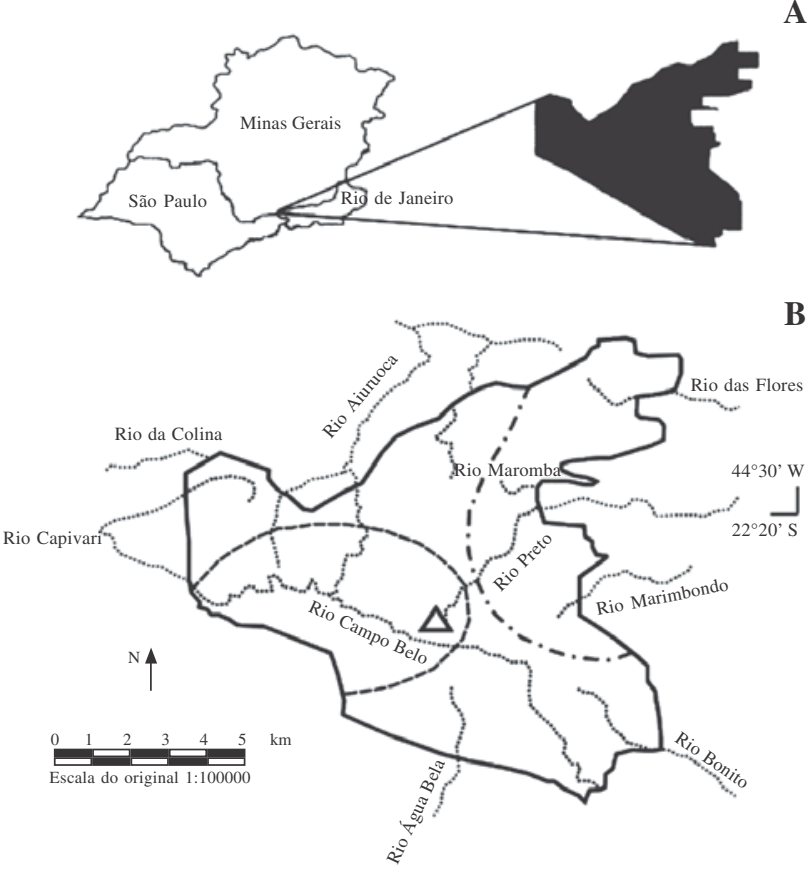

Figura 1. Localização do Parque Nacional de Itatiaia - Brasil: A) localização na região Sudeste; B) limites do Parque evidenciando as regiões de campos de altitude (---), Mata Atlântica (--*) e principais rios (...). ( $\triangle=$ Pico das Agulhas Negras).

Figure 1. Location of "Parque Nacional de Itatiaia" - Brazil: A) location in the southeastern region; B) limits of the Park showing the regions of highland grassfields (---), Atlantic Rainforest $\left(--^{-}\right)$and the main rivers (...). ( $\Delta=$ Peak of Agulhas Negras). 
Tabela 1. Localização e caracterização dos pontos de amostragem do "Parque Nacional de Itatiaia".

Table 1. Location and characteristics of sampling sites from "Parque Nacional de Itatiaia".

\begin{tabular}{|c|c|c|c|}
\hline $\begin{array}{l}\text { Site } \\
\text { number }\end{array}$ & Localização & $\begin{array}{l}\text { Altitude } \\
\quad(\mathrm{m})\end{array}$ & $\begin{array}{c}\text { Inclinação } \\
\left({ }^{\circ}\right)\end{array}$ \\
\hline & Mata Atlântica & & \\
\hline 1 & $\begin{array}{l}\text { Cachoeira do Rio Bonito, Trilha dos Três Picos; } \\
22^{\circ} 25^{\prime} 25^{\prime \prime} \text { S } 44^{\circ} 35^{\prime} 32^{\prime \prime} \text { W }\end{array}$ & 1640 & 4 \\
\hline 2 & $\begin{array}{l}\text { Córrego Simon (principal), Trilha dos Três Picos; } \\
22^{\circ} 25^{\prime} 53^{\prime \prime} \mathrm{S} 44^{\circ} 36^{\prime} 07^{\prime \prime} \mathrm{W}\end{array}$ & 1100 & 4 \\
\hline 3 & $\begin{array}{l}\text { Afluente do Córrego Simon, Trilha dos Três Picos; } \\
22^{\circ} 25^{\prime} 51^{\prime} \text { S } 44^{\circ} 36^{\prime} 27^{\prime} \text { W }\end{array}$ & 1090 & 8 \\
\hline 4 & $\begin{array}{l}\text { Córrego Maromba (afluente), } 100 \mathrm{~m} \text { abaixo da Cachoeira } \\
\text { Véu de Noiva; } 22^{\circ} 25^{\prime} 21^{\prime \prime} \mathrm{S}, 44^{\circ} 36^{\prime} 33^{\prime \prime} \mathrm{W} \text {. }\end{array}$ & 1190 & 2,5 \\
\hline 5 & $\begin{array}{l}\text { Rio Campo Belo (afluente), próximo ao Lago Azul. } \\
22^{\circ} 27^{\prime} 04^{\prime} \text { S } 44^{\circ} 36^{\prime} 35^{\prime \prime} \mathrm{W}\end{array}$ & 885 & 5 \\
\hline 6 & $\begin{array}{l}\text { Córrego Maromba (principal), } 50 \mathrm{~m} \text { abaixo da Cachoeira } \\
\text { Itaporani; } 22^{\circ} 25^{\prime} 24^{\prime \prime} \mathrm{S} 44^{\circ} 37^{\prime} 21^{\prime \prime} \mathrm{W}\end{array}$ & 1145 & 8 \\
\hline 7 & $\begin{array}{l}\text { Rio Campo Belo (afluente), trilha para parte alta; } \\
22^{\circ} 26^{\prime} 20^{\prime \prime} \mathrm{S} 44^{\circ} 37^{\prime} 51^{\prime \prime} \mathrm{W}\end{array}$ & 1180 & 6 \\
\hline & Campos de Altitude & & \\
\hline 8 & $\begin{array}{l}\text { Córrego das Agulhas Negras, trilha Aiuruoca-Rebouças; } \\
22^{\circ} 23^{\prime} 02^{\prime \prime} \mathrm{S} 44^{\circ} 40^{\prime} 02^{\prime \prime} \mathrm{W}\end{array}$ & 2490 & 2 \\
\hline 9 & $\begin{array}{l}\text { Rio Campo Belo, } 50 \mathrm{~m} \text { abaixo cachoeira; } \\
22^{\circ} 23^{\prime} 16^{\prime \prime} \mathrm{S} 44^{\circ} 40^{\prime} 31^{\prime \prime} \mathrm{W}\end{array}$ & 2340 & 5 \\
\hline 10 & $\begin{array}{l}\text { Rio Aiuruoca, próximo do fim da trilha Aiuruoca-Rebouças; } \\
22^{\circ} 22^{\prime} 09^{\prime \prime} \mathrm{S} 44^{\circ} 40^{\prime} 31^{\prime \prime} \mathrm{W}\end{array}$ & 2386 & 0,5 \\
\hline 11 & $\begin{array}{l}\text { Rio Campo Belo, (nascente), próximo à guarita do IBAMA; } \\
22^{\circ} 22^{\prime} 38^{\prime \prime} \mathrm{S} 44^{\circ} 41^{\prime} 37^{\prime \prime} \mathrm{W}\end{array}$ & 2355 & 4,5 \\
\hline 12 & $\begin{array}{l}\text { Ribeirão do Palmital, cachoeira próximo à guarita do IBAMA; } \\
22^{\circ} 22^{\prime} 31^{\prime \prime} \mathrm{S} 44^{\circ} 42^{\prime} 21^{\prime \prime} \mathrm{W}\end{array}$ & 2600 & 22 \\
\hline 13 & Córrego próximo à Pousada Alsene; $22^{\circ} 22^{\prime} 08^{\prime \prime} \mathrm{S} 44^{\circ} 42^{\prime} 30^{\prime \prime} \mathrm{W}$ & 2348 & 1,5 \\
\hline 14 & Córrego do Boiadeiro, Brejo da Lapa; 22²1’32” S 4444’14” W & 2150 & 1 \\
\hline
\end{tabular}

basicamente da delimitação de um trecho de 10 metros de extensão, subdividido em cotas iguais de 1 metro, ao longo das margens de cada rio/riacho a ser estudado. Neste trecho foram observadas, metro a metro, a presença/ausência de cada espécie, além de estimada a sua respectiva abundância relativa (cobertura percentual sobre o substrato através de estimativa visual). Os dados de cobertura foram computados como a média aritmética dos dez segmentos e extrapolados para todo o trecho (representativo da comunidade de macroalgas). Neste trabalho foi adotada a definição de macroalga proposta por Sheath \& Cole (1992): "tipicamente, macroalgas de ambientes lóticos são bentônicas e formam um talo maduro que é uma estrutura discreta e reconhecível a olho nu; a identificação microscópica é geralmente necessária e, freqüentemente, microalgas estão associadas com o talo". Adotou-se também a conceituação dos tipos morfológicos de macroalgas desses autores. A visualização das comunidades de macroalgas nos rios/riachos foi realizada com auxílio de observador subaquático com fundo de vidro (Necchi Júnior et al. 2000a).

Em laboratório os espécimes de macroalgas foram identificados, sempre que possível, até o nível espécie. Quando necessárias, identificações preliminares em campo foram realizadas (geralmente até o nível genérico) com auxílio de microscópio de campo Swift FM-31. A preservação dos espécimes foi feita em solução de formaldeído a 4\% (Necchi Júnior et al. 2000a). Conjuntos representativos de espécimes - testemunho foram incorporados no Herbário SJRP da UNESP, Campus de São José do Rio Preto (SJRP28345 a SJRP28372).

Foram anotadas, em cada ponto de amostragem, as seguintes variáveis ambientais: latitude, longitude, altitude, inclinação (do trecho), largura e profundidade médias dos trechos analisados, tipo de substrato, velocidade da correnteza, 
temperatura da água, turbidez, sombreamento, condutividade, $\mathrm{pH}$, oxigênio dissolvido e nutrientes (nitrogênio e fósforo totais, amônio, nitrato, ortofosfato, potássio e ferro), Demanda Química de Oxigênio (DQO) e cor. Foram registrados também dados referentes ao sombreamento, tipo de substrato e dimensões de cada trecho estudado. Os procedimentos e equipamentos para a medição das variáveis ambientais estão detalhadamente descritos nos trabalhos anteriores (Necchi Júnior et al. 2000a, 2000b, 2003).

As variáveis ambientais e biológicas têm sido referidas como seguindo distribuição normal em trabalhos anteriores (Necchi Júnior et al. 2000a, 2000b, 2003) e permitem a aplicação de testes paramétricos. O índice de diversidade utilizado foi o de Shannon e Wiener (Krebs 1989): $H^{\prime}=\Sigma s$ (pi. $\left.\log _{n} p_{i}\right)$, onde: $p_{i}=$ proporção (abundância $=\%$ cobertura) da espécie $i$ na comunidade; $i=1 ; s=$ número total de espécies na comunidade. As correlações das variáveis biológicas entre si e com as variáveis ambientais foi determinada através do coeficiente de correlação, $r$ de Pearson. A semelhança ou diferença entre as variáveis dos pontos de amostragens foram avaliadas pelo teste $t$ de Student (Zar 1999). A avaliação da relação conjunta das variáveis ambientais com as variáveis biológicas foi efetuada através de análise de regressão múltipla (Zar 1999). As comparações entre as classes de freqüência para as variáveis biológicas entre as regiões foram feitas através do teste do "qui-quadrado" (Zar 1999). Os testes estatísticos acima citados foram realizados com o uso dos pacotes estatísticos Statistica (versão 6.0, Statsoft).

As associações entre os pontos de amostragens, com base na composição de espécies, foram determinadas através da Análise de Grupamento utilizando-se o coeficiente de similaridade de Sorensen e média não-ponderada (UPGMA, Digby \& Kempton 1987). A Análise de Componentes Principais (ACP) foi aplicada a partir de matriz formada com os dados biológicos e ambientais referentes às duas regiões nos dois períodos estudados. A matriz foi padronizada pelo valor máximo ("ranging") e aplicou-se o coeficiente de correlação $r$ de Pearson. As variáveis ambientais selecionadas para esta análise foram as que apresentaram correlação significativa com as variáveis biológicas; foram eliminadas as variáveis que apresentaram forte colinearidade (Digby \& Kempton 1987): largura, profundidade, turbidez e nutrientes (nitrogênio e fósforo totais, amônio, nitrato, ortofosfato e ferro). Os testes estatísticos multivariados acima citados foram realizados com o uso do pacote estatístico MVSP "Multivariate Statistical Package" (versão 3.1, Kovach Computing Services). Os gráficos de distribuição da riqueza, abundância e diversidade das macroalgas nos pontos de amostragem foram realizados com o auxílio do software Microcal Origin (versão 5.0).

\section{Resultados}

Variáveis ambientais - Os rios/riachos estudados no PNI apresentaram algumas características gerais bem evidentes: valores relativamente baixos para temperatura $(14,5 \pm$ $\left.2,4^{\circ} \mathrm{C}\right)$, turbidez $(2,4 \pm 1,4 \mathrm{UTN}), \mathrm{pH}(6,0 \pm 0,5)$, condutividade $\left(10,4 \pm 4,1 \mu \mathrm{S} \mathrm{cm}^{-1}\right)$, cor $(0,5 \pm 0,3 \mathrm{~Hz})$, profundidade $(24,0 \pm 13,6 \mathrm{~cm})$ e nutrientes (nitrogênio total $0,27 \pm 0,16 \mathrm{mg} \mathrm{L}^{-1}$, amônio $0,11 \pm 0,05 \mathrm{mg} \mathrm{L}^{-1}$; nitrato $0,27 \pm 0,13 \mathrm{mg} \mathrm{L}^{-1}$; fósforo total $0,68 \pm 0,32 \mathrm{mg} \mathrm{L}^{-1}$, ortofosfato $0,46 \pm 0,21 \mathrm{mg} \mathrm{L}^{-1}$; potássio $0,63 \pm 0,51 \mathrm{mg} \mathrm{L}^{-1}$; ferro $\left.0,15 \pm 0,03 \mathrm{mg} \mathrm{L}^{-1}\right)$; moderados de velocidade da correnteza $\left(55 \pm 42 \mathrm{~cm} \mathrm{~s}^{-1}\right)$ e saturação de oxigênio $(57,1$ $\pm 6,0 \%$; e valores elevados de DQO $\left(15,4 \pm 6,3 \mathrm{mg} \mathrm{l}^{-1}\right)$. As larguras dos pontos de amostragem foram muito variadas $(0,5-4,1 \mathrm{~m})$, enquanto que doze dos quatorze pontos de amostragem tiveram substrato rochoso (rocha contínua ou matacões).

Foram encontradas diferenças significativas $(p<0,01)$ entre as duas regiões do PNI para as seguintes variáveis: condutividade $(t=3,2), \mathrm{pH}(t=3,6)$, cor $(t=4,1)$ e saturação de oxigênio $(t=2,9)$, com os maiores valores observados nos riachos de MA. Foram verificadas diferenças significativas $(p<0,05)$ entre as duas estações do ano (considerando-se as duas regiões conjuntamente) para as seguintes variáveis: temperatura $(t=3,7)$, condutividade $(t=3,6), \mathrm{pH}(t=2,3)$, velocidade da correnteza $(t=2,3)$ e DQO $(t=2,9)$; os valores mais elevados de temperatura, velocidade e DQO ocorreram no verão, enquanto que os de condutividade e $\mathrm{pH}$ foram mais altos no inverno. Os testes de correlação revelaram valores significativos $(p<0,05)$ entre as seguintes variáveis: temperatura $\mathrm{x}$ oxigênio dissolvido $(r=0,69)$; pH x condutividade $(r=0,55) ; \mathrm{pH} \times$ DQO $(r=0,48)$; cor x oxigênio dissolvido $(r=0,51)$.

Estrutura das comunidades de macroalgas do PNI Foram encontrados 29 táxons infra-genéricos de macroalgas nos 14 pontos de coleta do PNI durante as duas estações do ano, incluindo cinco grupos vegetativos (Batrachospermum sp., Mougeotia spp., Oedogonium spp., Spirogyra spp. e Zygnema spp.) (tabela 2). Cyanophyta e Chlorophyta foram os grupos predominantes (41 e 38\% dos táxons, respectivamente), com pouca representatividade de Rhodophyta e Heterokontophyta (14 e 7\%, respectivamente). Chlorophyta foi melhor representada em CA do que em MA (dez x três táxons), enquanto que representantes de Heterokontophyta ocorreram apenas em MA. O gênero Stigonema foi o melhor representado, com quatro espécies (uma delas ainda não identificada), seguido de Phormidium, com três espécies (tabela 2). As espécies mais frequientes foram Stigonema robustum (oito registros) e Klebsormidium fluitans (seis registros); por outro lado, 12 táxons tiveram um único registro de ocorrência (tabelas 2-3). Foi observada a seguinte proporção dos tipos morfológicos entre os táxons infra-genéricos encontrados: filamento livre (35\%), 
Tabela 2. Táxons encontrados no Parque Nacional de Itatiaia nos dois períodos de amostragem. Pontos de amostragem 1-7: região de Mata Atlântica; Pontos de amostragem 8-14: região de campos de altitude.

Table 2. Taxa surveyed in "Parque Nacional de Itatiaia" at both sampling seasons. Sampling sites 1-7: Atlantic Rainforest region; sampling sites 8-14: highland grassfields region.

\begin{tabular}{|c|c|c|c|c|c|c|c|c|c|c|c|c|c|c|}
\hline \multirow{3}{*}{ Táxons } & \multicolumn{14}{|c|}{ Pontos de Amostragem } \\
\hline & \multicolumn{7}{|c|}{ Mata Atlântica (MA) } & \multicolumn{7}{|c|}{ Campos de altitude (CA) } \\
\hline & 1 & 2 & 3 & 4 & 5 & 6 & 7 & 8 & 9 & 10 & 11 & 12 & 13 & 14 \\
\hline
\end{tabular}

\section{CYANOPHYTA}

Chamaesiphon curvatus Nordst.

Dichothrix baueriana (Grun.) Born. \& Flah.

Microcoleus subtorulosus Gom.

Phormidium retzii (C. Ag.) Gom.

P. schroederi (Borge) Anagn. \& Komárek

P. willei (Gardn.) Anagn. \& Komárek

Scytonema cf. arcangeli Born. \& Flah.

Stigonema cf. informe Born. \& Flah.

S. mamilosum (Lyngbye) Born. \& Flah.

$S$. robustum Gardn.

Stigonema sp.

Tolypothrix distorta Born. \& Flah.

Total Cyanophyta (12)

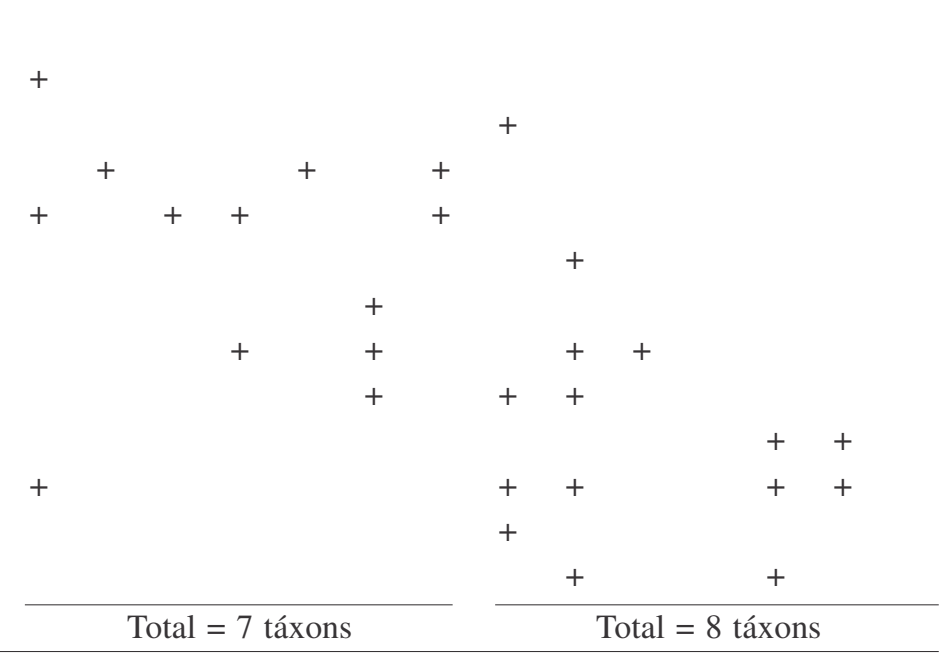

\section{CHLOROPHYTA}

Draparnaldia glomerata (Vauch.) C. Ag.

Klebsormidium fluitans (Gay) Lokh.

Microspora tumidula Haz.

M. wittrocki (Wille) Lag.

Mougeotia spp. (estéreis)

Nitella furcata Bruz. subsp. mucronata (A. Br.)

Wood

Oedogonium spp. (estéreis)

Spirogyra spp. (estéreis)

Stigeoclonium subsecundum (Kütz.) Kütz.

Tetraspora lubrica (Roth) C. Ag.

Zygnema spp. (estéreis)

Total Chlorophyta (11)

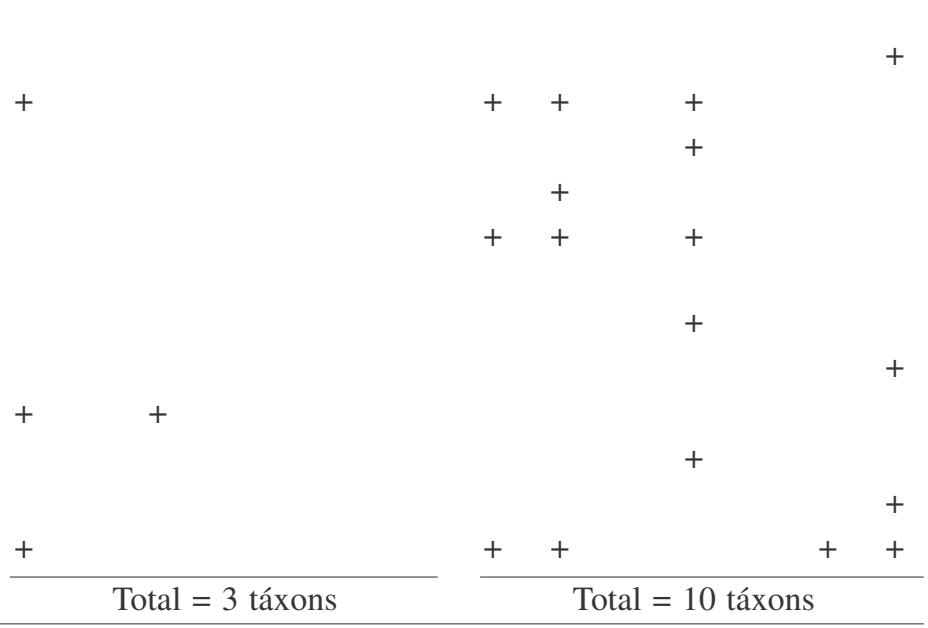

\section{HETEROKONTOPHYTA}

Fragilaria javanica Hust.

Melosira varians C. Ag.

Total Heterokontophyta (2)

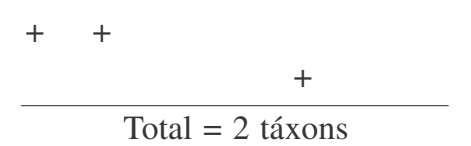

Total $=0$ táxons

\section{RHODOPHYTA}

Batrachospermum atrum (Huds.) Harv.

Batrachospermum sp. (estéril)

Hildenbrandia angolensis West \& West

Estágio 'Chantransia’ de Batrachospermum spp.

Total Rhodophyta (4)

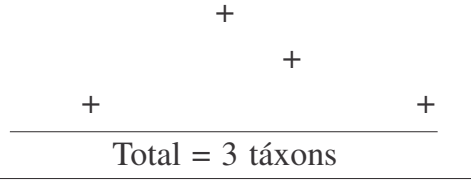

Total do Parque (29)

(15)

Total = 1 táxon 


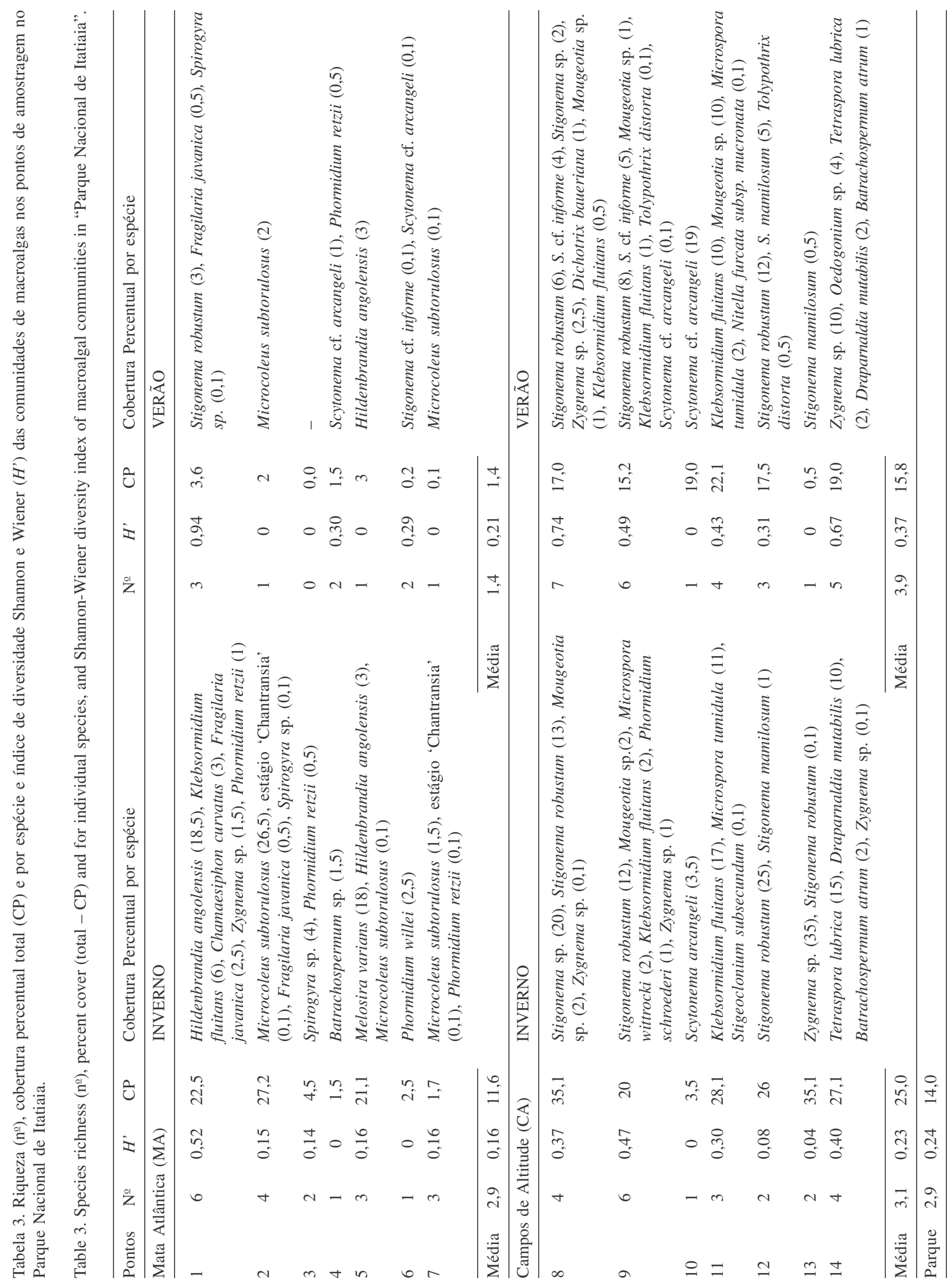


emaranhado (24\%), tufo (14\%), incrustante (14\%), filamento gelatinoso (10\%) e colônia gelatinosa (3\%).

Os dados biológicos variaram da seguinte maneira (tabela 3, figura 2): os valores de cobertura percentual e índice de diversidade de Shannon-Wiener $\left(H^{\prime}\right)$ por ponto
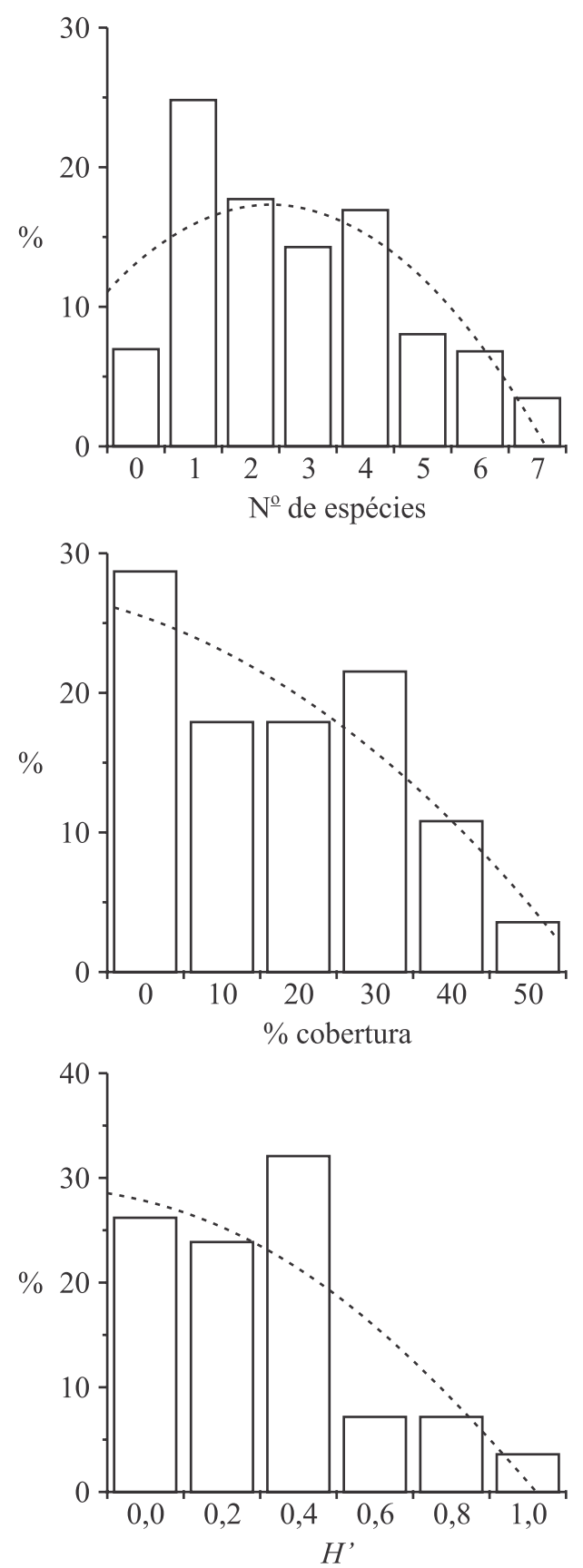

Figura 2. Distribuição de freqüência dos dados biológicos das comunidades de macroalgas de ambientes lóticos do Parque Nacional de Itatiaia. $N=28$.

Figure 2. Frequency distribution of biological data of macroalgal communities from "Parque Nacional de Itatiaia". $N=28$. de amostragem variaram de 0 a $35 \%(14,0 \% \pm 12,5 \%)$ e 0 a $0,94(0,24 \pm 0,26)$, respectivamente. Os valores de riqueza de espécies variaram de 0 a 7 por ponto de amostragem $(2,9 \pm 2,0)$. O teste de "Qui-quadrado" revelou diferenças significativas $(p<0,01)$ entre as duas regiões estudadas (MA e CA) para as variáveis biológicas: número de espécies $\left(\chi^{2}=22,4\right) ; H^{\prime}\left(\chi^{2}=23,0\right)$; e cobertura $\left(\chi^{2}=56,7\right)$, com os maiores valores médios em CA (tabela 3). Correlações significativas $(p<0,05)$ foram encontradas entre as variáveis biológicas com algumas variáveis ambientais: cobertura percentual correlacionouse negativamente com temperatura $(r=-0,62)$ e saturação de oxigênio ( $r=-0,47)$, e positivamente com ferro $(r=0,46)$; $H$ ' esteve negativamente correlacionada com $\mathrm{pH}(r=-0,48)$ e positivamente com DQO $(r=0,47)$; riqueza de espécies correlacionou-se negativamente com temperatura $(r=-0,63)$. Cobertura e $H$ ' correlacionaram-se positivamente com riqueza ( $r=0,53$ e 0,80 , respectivamente). A análise de regressão múltipla mostrou que: a) 39,2\% da variação da abundância das espécies foi explicada pelas variações de temperatura; b) $54,8 \%$ da variação da diversidade foi explicada por DQO e pH (44,8\% e 10\%); c) 35,5\% da variação da riqueza de espécies foi explicada pelas variações de temperatura.

A análise de grupamento das comunidades de macroalgas do PNI (dados não apresentados) revelou baixas similaridades entre os grupos formados $(<50 \%)$ e não agrupou os pontos das duas regiões estudadas (MA e CA). Pequenos grupos foram formados devido à ocorrência de apenas uma espécie em comum. Além disso, das 29 espécies inventariadas no PNI, 15 ocorreram exclusivamente em MA e 19 em CA; apenas cinco espécies foram coletadas em ambas as regiões (tabela 2 ).

A ACP (tabela 4, figura 3) mostrou nítida separação entre os pontos das duas regiões (MA e CA). O eixo 1 separou os pontos de MA (metade esquerda), correlacionados positivamente com sombreamento e $\mathrm{pH}$ e negativamente com altitude, daqueles de CA (metade direita) com tendência inversa. No eixo 2 foram separadas as duas estações do ano: inverno - estação seca (metade superior), correlacionadas positivamente com potássio e negativamente com temperatura e DQO; verão - estação chuvosa (metade inferior) com tendência inversa. Os pontos 1i, $10 \mathrm{i}$ e $1 \mathrm{v}$ foram exceções porque se posicionaram relativamente distantes dos respectivos grupamentos devido a valores extremos para variáveis biológicas ou ambientais.

\section{Discussão}

O número de táxons infra-genéricos encontrado (29) foi relativamente alto em relação aos dados de outros 


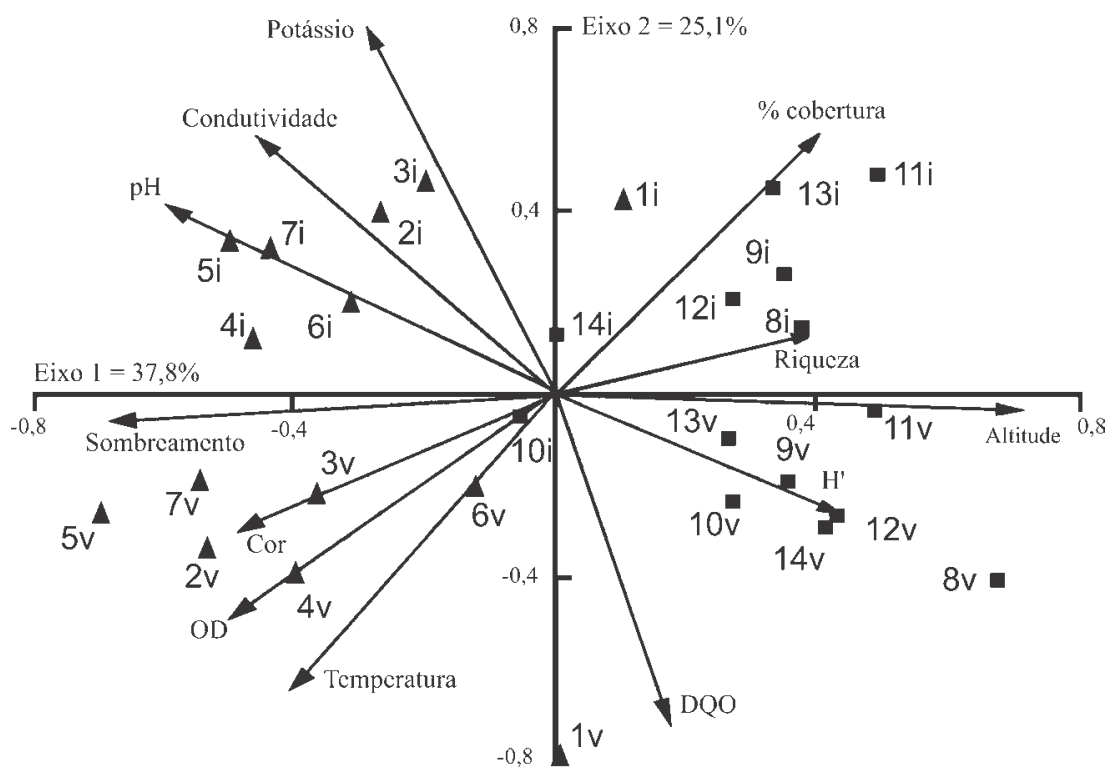

Figura 3. Representação gráfica da Análise dos Componentes Principais dos pontos de amostragem nas duas estações do ano com base nas variáveis ambientais e biológicas do Parque Nacional de Itatiaia. (DQO = Demanda Química de Oxigênio; $H^{\prime}$ = Índice de diversidade de Shannon-Wiener; OD = Oxigênio Dissolvido. $2 \%$ = pontos de amostragem da região de Mata Atlântica; \% = pontos de amostragem da região de campos de altitude; i e v após os números dos pontos de amostragem indicam, respectivamente, inverno e verão).

Figure 3. Principal Component Analysis results of sampling sites at the two sampling seasons on the basis of environmental and biological data of "Parque Nacional de Itatiaia". (DQO = Chemical Oxygen Demand; $H$ ' = Shannon-Wiener diversity index; OD = Dissolved Oxygen; $2 \%=$ sampling sites from Atlantic Rainforest; $\%=$ sampling sites from highland grassfields; $i$ and $v$ after the number of sampling sites indicate, winter and summer, respectively).

Tabela 4. Resultados da Análise de Componentes Principais (ACP) incluindo-se as variáveis (biológicas e ambientais) e correlação de cada variável com os dois primeiros eixos. As variáveis significativas estão destacadas em negrito.

Table 4. Principal Component Analysis results including variables (biological and environmental) and correlation of each variable with the first two axes. Significant variables are indicated with boldface.

\begin{tabular}{lcc}
\hline Variáveis/resultados & Eixo 1 & Eixo 2 \\
\hline Autovalores & 4,5 & 3,0 \\
Variação explicada (\%) & 37,8 & 25,1 \\
Variação cumulativa (\%) & 37,8 & 62,9 \\
Altitude & $\mathbf{0 , 4 2 8}$ & $-0,020$ \\
Temperatura & $-0,243$ & $\mathbf{- 0 , 3 8 9}$ \\
Condutividade & $-0,275$ & 0,338 \\
pH & $\mathbf{- 0 , 3 5 0}$ & 0,233 \\
Oxigênio Dissolvido & $-0,299$ & $-0,294$ \\
Cor & $-0,296$ & $-0,177$ \\
Potássio & $-0,173$ & $\mathbf{0 , 4 7 9}$ \\
DQO & 0,102 & $\mathbf{- 0 , 4 2 7}$ \\
Sombreamento & $\mathbf{- 0 , 3 9 4}$ & $-0,033$ \\
Riqueza & 0,241 & 0,081 \\
Diversidade $\left(H^{\prime}\right)$ & 0,263 & $-0,157$ \\
Cobertura percentual & 0,243 & 0,343 \\
\hline
\end{tabular}

estudos, considerando-se o esforço de amostragem (14 pontos): 2,1 espécies/ponto de amostragem. Este valor é próximo ao maior já reportado para comunidades de macroalgas lóticas (Necchi Júnior et al. 2003): 30 espécies em 12 pontos de amostragem (2,5). Outros estudos realizados em diversas regiões do mundo e do Brasil encontraram valores bem abaixo desses: 40-67 espécies em 40-52 pontos de amostragem - 0,8-1,3 (Sheath et al. 1986, 1989, Branco \& Necchi Júnior 1996a, Necchi Júnior et al. 1997). Por outro lado, deve-se ressalvar que há tendência de diminuição do número de novas ocorrências com o aumento do número de pontos de amostragem, pois ocorre repetição de espécies (Branco \& Necchi Júnior 1996a, Sheath \& Cole 1992, Necchi Júnior et al. 2000a).

Os grupos predominantes (Cyanophyta e Chlorophyta) concordam parcialmente com levantamentos anteriores (Sheath \& Burkholder 1985, Biggs 1990, Sheath \& Cole 1992, Sheath et al. 1996, Necchi Júnior et al. 1997, Necchi Júnior et al. 2000a), exceto que o grupo melhor representado têm sido tipicamente Chlorophyta (38\% neste trabalho x 35\%-37\% nos anteriores), seguido de Cyanophyta (41\% x 24\%-35\%). Porém, esses dados são muito semelhantes àqueles em estudo anterior em região 
de campos de altitude do Parque Nacional da Serra da Canastra (Necchi Júnior et al. 2003, 40\% Cyanophyta, $36,5 \%$ Chlorophyta); mas em menor grau pela baixa proporção de Rhodophyta (14\% x 23,5\%). A predominância de Cyanophyta pode ser atribuída à adaptação a ambientes com diferentes níveis de sombreamento (sombreados em MA e abertos em CA), bem como às condições oligotróficas prevalecentes nos riachos do PNI, nas quais as formas heterocitadas (58\% das espécies do grupo e $87,5 \%$ das espécies presentes em CA), podem ter vantagens adaptativas. Esses dados corroboram aqueles reportados por Necchi Júnior et al. (2003) de ocorrência exclusiva de espécies heterocitadas, atribuída presumivelmente à baixa disponibilidade de nitrogênio inorgânico. As proporções dos tipos morfológicos foram bastante distintas daquelas reportadas em estudos anteriores (Sheath \& Cole 1992, Necchi Júnior et al. 2000a, 2003), notadamente a freqüência bem maior de filamentos livres (35\% x 8\%-15\%) e muito menor de emaranhados (24\% x $42-52 \%$ ).

A temperatura da água foi a variável ambiental com maior e mais consistente influência sobre as variáveis biológicas (riqueza, abundância e diversidade de espécies) relacionadas à estrutura das comunidades de macroalgas do PNI. A temperatura tem sido reportada como variávelchave em estudos ecológicos de algas bentônicas de ecossistemas aquáticos continentais em diferentes escalas espaciais e temporais (Denicola 1996 e referências). A influência da temperatura neste estudo provavelmente está relacionada com a amostragem em gradiente de altitude e vegetação, com evidentes reflexos nas características térmicas dos corpos d'água em cada região (MA e CA) e conseqüientes efeitos dessa variação de temperatura sobre a estrutura das comunidades de macroalgas. A média de temperatura observada para o PNI $\left(14,5^{\circ} \mathrm{C}\right)$ foi a mais baixa já reportada em estudos de macroalgas de ambientes lóticos de regiões brasileiras (Necchi Júnior et al. 2000a, 2000b, 2003). Este fato é relevante por tratar-se de área montanhosa situada em região tropical e reforça a suposição anterior sobre o esperado efeito do gradiente de temperatura.

Em comparação com outras regiões brasileiras semelhantes, verificou-se que das 19 espécies da região de CA do PNI, apenas três (16\%) foram comuns com outra de campos de altitude previamente amostrada (Necchi Júnior et al. 2003). Em comparação com outras regiões do mundo, a flora de CA do PNI apresentou três espécies em comum com região andina da Bolívia (McClintic et al. 2003) e cinco (26\%) com região de tundra da América do Norte (Sheath et al. 1996). Com relação à região de MA, seis espécies (40\%) ocorreram na flora desse bioma no Estado de São Paulo (Branco \& Necchi Junior 1996a, b). Em termos mundiais, a flora de MA do PNI teve duas espécies comuns (13\%) com região de floresta tropical montana da Bolívia (McClintic et al. 2003) e cinco (33\%) com floresta tropical da América do Norte (Sheath \& Cole 1992). Estes resultados indicam baixa similaridade da flora do PNI, particularmente para a região de campos de altitude, com outras regiões estudadas do país e do mundo.

Os valores encontrados situaram-se dentro das amplitudes reportadas para riqueza de espécies $(2,9 \mathrm{x}$ 1,8-5,6) e abundância (14\% x 6\%-43\% de cobertura) em outros estudos (Sheath \& Cole 1992, Branco \& Necchi Júnior 1996b, 1998, McClintic et al. 2003, Necchi Júnior et al. 2000a, 2003). Os padrões gerais de distribuição descritos para comunidades de macroalgas lóticas (Sheath et al. 1986, Sheath \& Cole 1992, Necchi Júnior et al. 2000a) foram em geral corroborados pelos dados gerais dos rios/riachos do PNI, a saber: distribuição em mosaico, evidenciada pela correlação positiva entre riqueza, abundância e diversidade de espécies (as comunidades mais abundantes são também as mais diversas); forte dominância por poucas espécies, verificada pela dominância quantitativa (em termos de cobertura percentual nos pontos de amostragem) ou predominância qualitativa (em termos de número de registros de ocorrência na região de estudo) de uma ou poucas espécies nos pontos de amostragem). Em síntese, este estudo confirma que tais padrões parecem ser aplicáveis universalmente às comunidades de macroalgas lóticas.

Agradecimentos - este projeto foi apoiado pelo CNPq (Proc. 304067/2003-2 e 500252/2003-3) e FAPESP (02/04141-3). Agradecemos a colaboração no trabalho de campo de Emerson N.M. Moura e de laboratório de Maria Helena Carabolante. Este projeto refere-se à licença do IBAMA no. 169/03.

\section{Referências bibliográficas}

BICUDO, C.E.M. \& BICUDO, R.M.T. 1969. Algas da Lagoa das Prateleiras, Parque Nacional do Itatiaia, Brasil. Rickia 4:1-40.

BIGGS, B.J.F. 1990. Periphyton communities and their environments in New Zealand rivers. New Zealand Journal of Marine and Freshwater Research 24:367-386.

BIGGS, B.J.F. \& PRICE, G.M. 1987. A survey of filamentous algal proliferations in New Zealand rivers. New Zealand Journal of Marine and Freshwater Research 21:175-191.

BRANCO, C.C.Z. \& NECCHI JÚNIOR, O. 1996a. Survey of stream macroalgae of eastern Atlantic Rainforest of São Paulo State, southeastern Brazil. Algological Studies 80:35-57. 
BRANCO, C.C.Z. \& NECCHI JÚNIOR, O. 1996b. Distribution of stream macroalgae in the eastern Atlantic Rainforest of São Paulo State, southeastern Brazil. Hydrobiologia 333:139-150.

BRANCO, L.H.Z. \& NECCHI JÚNIOR, O. 1997. Seasonality of macroalgae in three tropical drainage basins in São Paulo State, southeastern Brazil. Archiv für Hydrobiologie 141:75-91.

BRANCO, L.H.Z. \& NECCHI JÚNIOR, O. 1998. Distribution of macroalgae in three tropical drainage basins in São Paulo State, southeastern Brazil. Archiv für Hydrobiologie 142:241-256.

CALLISTO, M., MORENO, P. \& BARBOSA, F.A.R. 2001. Habitat diversity and benthic functional trophic groups at Serra do Cipó, southeast Brazil. Revista Brasileira de Biologia 61:259-266.

DENICOLA, D.M. 1996. Periphyton responses to temperature at different ecological levels. In Algal ecology: freshwater benthic ecosystems, (R.J. Stevenson, M.L. Bothwell \& R.L. Lowe, eds.), Academic Press, San Diego, p.149181.

DIGBY, P.G.N. \& KEMPTON. R.A. 1987. Multivariate analysis of ecological communities. Chapman and Hall, London.

ENTWISLE, T.J. 1989. Macroalgae in Yarra River basin: flora and distribution. Proceedings of the Royal Society of Victoria 101:1-76.

GALDEAN, N., CALLISTO, M. \& BARBOSA, F.A.R. 2001. Biodiversity assessment of benthic macroinvertebrates in altitudinal lotic ecosystems of Serra do Cipó (MG, Brazil). Revista Brasileira de Biologia 61:239-248.

GUSTINA, G.W. \& HOFFMANN, J.P. 2000. Periphyton dynamics in a subalpine mountain stream during winter. Arctic, Antarctic and Alpine Research 32:127-134.

HIEBER, M., ROBINSON, C.T., RUSHFORTH, S.R. \& UEHLINGER, U. 2001. Algal communities associated with different alpine stream types. Arctic, Antarctica and Alpine Research 33:447-456.

HOLMES, N.T.H. \& WHITTON, B.A. 1981. Phytobenthos of River Tees and its tributaries. Freshwater Biology 11:43-60.

IBAMA (Instituto Brasileiro do Meio Ambiente e dos Recursos Naturais Renováveis). 1994. Plano de ação emergencial para o Parque Nacional de Itatiaia. IBAMA, Brasília.

JOHANSSON, C. 1982. Attached algal vegetation in running water of Jämtland, Sweden. Acta Phytogeographica Suecica 74:1-84.

JOHN, D.M. \& MOORE, J.A. 1985. Observations on the phytobenthos of the freshwater Thames, I: The environment, floristic composition and distribution of the macrophytes (principally macroalgae). Archiv für Hydrobiologie 102:435-459.

KAWECKA, B. 1980. Sessile algae in European mountain streams 1 . The ecological characteristics of communities. Acta Hydrobiologica 22:361-420.
KAWECKA, B. 1981. Sessile algae in European mountain streams 2. Taxonomy and autoecology. Acta Hydrobiologica 23:17-46.

KAWECKA, B. \& ELORANTA, P. 1987. Communities of sessile algae in some small streams of Central Finland: comparison of algae of the high mountains of Europe and those in northern region. Acta Hydrobiologica 29:403-315.

KREBS, C.J. 1989. Ecological methodology. Harper \& Row, New York.

MCCLINTIC, A.S., CASAMATTA, D.A. \& VIS, M.L. 2003. A survey of algae from montane cloud florest and alpine streams in Bolivia: macroalgae and associated microalgae. Nova Hedwigia 76:363-379.

MEDVEDEVA, L.A. 2001. Biodiversity of aquatic algal communities in the Sikhote-Alin biosphere reserve (Russia). Cryptogamie, Algologie 22:65-100.

MEEGAN, S.K. \& PERRY, S.A. 1996. Periphyton communities in headwater streams of different water chemistry in the Central Appalachian Mountains. Journal of Freshwater Ecology 11:247-256.

MENDES, R.S. \& BARBOSA, F.A.R. 2002. Efeito do enriquecimento "in situ" sobre a biomassa da comunidade perifítica de um córrego de altitude da Serra do Cipó (MG). Acta Limnologica Brasiliensia 14:77-86.

MOSISCH, T.D. \& BUNN, S.E. 1997. Temporal patterns of rainforest stream epilithic algae in relation to flowrelated disturbance. Aquatic Botany 58:181-193.

NECCHI JÚNIOR, O., BRANCO, C.C.Z. \& BRANCO, L.H.Z. 2000a. Distribution of stream macroalgae in São Paulo State, southeastern Brazil. Algological Studies 97:43-57.

NECCHI JÚNIOR, O., BRANCO, L.HZ. \& BRANCO, C.C.Z. 2000b. Características limnológicas do Alto Rio São Francisco, Parque Nacional da Serra da Canastra, Minas Gerais. Acta Limnologica Brasiliensia 12:1122.

NECCHI JÚNIOR, O., BRANCO, L.H.Z. \& BRANCO, C.C.Z. 2003. Ecological distribution of stream macroalgal communities from a drainage basin in the Serra da Canastra National Park, Minas Gerais, Southeastern Brazil. Revista Brasileira de Biologia 63:1-12.

NECCHI JÚNIOR, O., BRANCO, C.C.Z., SIMÕES, R.C.G. \& BRANCO, L.H.Z. 1995. Distribution of stream macroalgae in the northwest region of São Paulo State, southeastern Brazil. Hydrobiologia 299:219-230.

NECCHI JÚNIOR, O., PASCOALOTO, D., BRANCO, C.C.Z. \& BRANCO, L.H.Z. 1997. Stream macroalgal flora from the northwest region of São Paulo State, southeastern Brazil. Algological Studies 84:91-112.

ORMEROD, S.J., RUNDLE, S.D., WILKINSON, S.M., DALY, G.P. \& JUTTNER, I. 1994. Altitudinal trends in the diatoms, bryophytes, macroinvertebrates and fish of a Nepalese river system. Freshwater Biology 32:309-322. 
PASCOALOTO, D. 2001. Características ambientais de cinco igarapés de terra-firme em reservas florestais no Estado do Amazonas e sua relação com Batrachospermum cayennense (Batrachospermaceae, Rhodophyta). Acta Amazonica 31:597-606.

PFISTER, P. 1992. Artenspektrum des Algenaufwuchses in 2 Tiroler Bergbachen - Teil 1: Cyanophyceae, Chrysophyceae, Chlorophyceae, Rhodophyceae. Algological Studies 65:43-61.

PFISTER, P. 1993. Seasonality of macroalgal distribution patterns within the reach of a gravel stream (Isar, Tyron, Austria). Archiv für Hydrobiologie 129:89-107.

SHEATH, R.G. \& BURKHOLDER, J.M. 1985. Characteristics of softwater streams in Rhode Island. II: Composition and seasonal dynamics of macroalgal communities. Hydrobiologia 128:109-118.

SHEATH, R.G. \& COLE, K.M. 1992. Biogeography of stream macroalgae in North America. Journal of Phycology 28:448-460.

SHEATH, R.G., HAMILTON, P.B., HAMBROOK, J.A. \& COLE, K.M. 1989. Stream macroalgae of the eastern boreal forest region of North America. Canadian Journal of Botany 67:3353-3362.

SHEATH, R.G., MORISON, M.O., KORCH, J.E., KACZMARCZYK, D. \& COLE, K.M. 1986. Distribution of stream macroalgae in south-central Alaska. Hydrobiologia 135:259-269.
SHEATH, R.G. \& MÜLLER, K.M. 1997. Distribution of stream macroalgae in four high Arctic drainage basins. Arctic 50:355-364.

SHEATH, R.G., VIS, M.L., HAMBROOK, J.A. \& COLE, K.M. 1996. Tundra stream macroalgae of North America: composition, distribution and physiological adaptations. Hydrobiologia 336:67-82.

UEHLINGER, U. 1991. Spatial and temporal variability of the periphyton biomass of a prealpine river (Necker, Switzerland). Archiv für Hydrobiologie 123:219-231.

VAVILOVA, V.V. \& LEWIS JUNIOR, W.M. 1999. Temporal and altitudinal variations in the attached algal of mountain streams in Colorado. Hydrobiologia 390:99-106.

VIS, M.L., SHEATH, R.G., HAMBROOK, J.A. \& COKE, K.M. 1994. Stream macroalgae of the Hawaiian islands: a preliminary study. Pacific Science 48:175-187.

WARD, J.V. 1986. Altitudinal zonation in a Rocky Mountain stream. Archir für Hydrobiologie, Supplement 74:133-199.

WARD, J.V. 1994. Ecology of alpine streams. Freshwater Biology 32:277-294.

WEHR, J.D. 1981. Analysis of seasonal succession of attached algae in a mountain stream, the North Alouette River, British Columbia. Canadian Journal of Botany 59:1465-1474.

WHITTON, B.A. 1984. Ecology of European Rivers. Blackwell Science Publishers, Oxford.

ZAR, , J.H. 1999. Biostatistical analysis, $4^{\text {th }}$ ed. Prentice Hall, Upper Saddle River. 